\title{
Detector chain calibration strategy for the Euclid Flight IR H2RGs
}

\author{
Barbier R. ${ }^{a}$, Buton C. ${ }^{\mathrm{a}}$, Clemens J-C. ${ }^{\mathrm{b}}$, Conversi L. ${ }^{\mathrm{d}}$, Ealet A. ${ }^{\mathrm{b}}$, Ferriol S. ${ }^{\mathrm{a}}$, Fornari F. ${ }^{\text {e }}$, Gillard \\ W. ${ }^{b}$, Kohley R. ${ }^{d}$, Kubik B. ${ }^{a}$, Rosset C. ${ }^{c}$, Secroun A. ${ }^{\text {, Serra B. }}{ }^{b}$, Smadja G. ${ }^{a}$, and Zoubian J. ${ }^{b}$

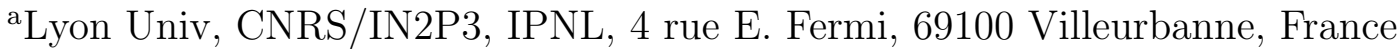 \\ ${ }^{\mathrm{b}}$ Aix-Marseille Univ, CNRS/IN2P3, CPPM, Marseille, France \\ ${ }^{\mathrm{c}}$ Paris Diderot Univ, CNRS/IN2P3, APC, Bat. Condorcet, 75205 Paris, France \\ ${ }^{\mathrm{d} E S A C}$, ESA, Camino Bajo del Castillo, 28692 Villanueva de la Canada, Spain \\ eIstituto Nazionale di Fisica Nucleare, Sezione di Bologna, Via Irnerio 46, Bologna 40126, Italy
}

\begin{abstract}
Euclid is an ESA mission to map the geometry of the Dark Universe with a planned launch date in $2021 .^{1}$ Two primary cosmological probes, weak gravitational lensing and baryonic acoustic oscillations, are implemented through a VISible imager (VIS) and a Near-Infrared Spectrometer and Photometer (NISP). The ground characterization of the NISP Flight Sensor Chip Systems (SCS) followed by the pixel response calibration aims to produce all informations to correct and control the accuracy of the signal. This work reports on the ground characterization of the NISP detector chain. The detector and electrical effects are likely to generate statistical fluctuations and systematic errors on the final flux measurement. The analysis strategies to maintain the pixel relative response accuracy within $1 \%$ is proposed in this work. The Euclid NISP test flow is presented and the main concerns of the detector chain calibration, such as non-linearity, charge trapping and de-trapping are discussed on the basis of the analysis of the flight detectors characterization data.
\end{abstract}

Keywords: Euclid, NISP, HgCdTe, H2RG, non-linearity, latency, reciprocity failure, trapping

\section{INTRODUCTION}

The NISP focal plane is composed of a 4x4 mosaic of Sensor Chip Assemblies (SCAs). The SCAs are $16 \mathrm{HgCdTe}$ detectors produced by Teledyne Imaging Sensors. They are commonly named H2RG. ${ }^{2}$ The cut-off has been tuned to $2.3 \mu \mathrm{m}$. The readout electronic of the SCAs are 16 SIDECARs called in NISP, the Sensor Chip Electronics (SCE). ${ }^{3}$ The ground characterization performed by the NISP detector team was dedicated to the calibration of the pixel response. The NISP characterization and data analysis aim to produce pixel maps for different purposes:

- to verify the detector chain performances,

- to feed the internal detector chain calibration pipeline,

- to feed the processing pipeline of the Science Ground Segment,

- to feed the detector chain simulation.

The signal extracted from the NISP detector chain is obtained by measuring a non-destructive readout of the pixel. This readout mode is called Sampling up the Ramp (SUTR). The in-flight readout mode of the NISP is a particular mode of SUTR: the MultiACCumulation (MACC) mode which minimizes the on-board processing keeping the ability to produce a Quality Factor of the signal sent to the ground. The MACC readout mode is illustrated in figure 1.

Further author information: Rémi Barbier. E-mail: rbarbier@ipnl.in2p3.fr, Telephone: 33472431222 


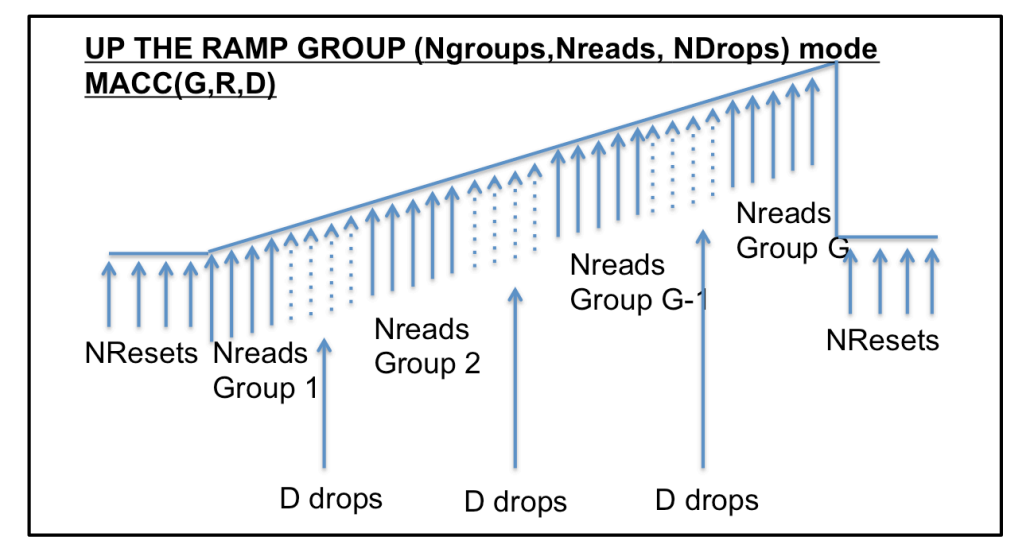

Figure 1. Scheme of the Multi ACCumulation (MACC) readout mode for one ramp. The spectroscopic observation with an effective integration time of $565 \mathrm{~s}$ is read out with a $\operatorname{MACC}(15,16,11)$. Photometric observation could be either a Fowler, $\operatorname{MACC}(2,16, \mathrm{D})$ or a $\operatorname{MACC}(\mathrm{G}, 16, \mathrm{D})$ with $\mathrm{G}=3,4$ or 5 . The frame time is $1.4548 \mathrm{~s}$

This conference paper presents in section 2 the main requirements on the detector chain calibration accuracy and the products (pixel maps) that the NISP detector team is foreseen to deliver from the ground characterization campaign. Section 3 deals with the description of the test flow. First, the content of the characterization is described and then the execution of the test flow performed at CPPM in 2017 and 2018 is summarized. Section 4 deals with the main objective of this paper, namely the pixel response calibration strategy. Section 4.1 presents the methods while section 4.2 shows preliminary results obtained from Flight Run data.

\section{NIR DETECTOR CHAIN CALIBRATION ACCURACY REQUIREMENTS}

\subsection{Requirements}

The main motivation for a precise detector chain error measurement is the control of the systematics on the fluxes of the sources. The detector chain error is defined as the systematic error (Req. 1\%) on the correction of the pixel response. The correction functions aims to rectify the value of the slope of the ramp modified by detector and electronic effects. These effects include bias drift, dark current, non-linearity in the charge and the voltage domains, trapping (persistence) and de-trapping of charge carriers, electronic cross talk between readout channels, inter-pixel capacitance contribution from the neighbour pixels, and intra-pixel variations.

Operable pixels From the survey efficiency flows down the requirement on the percentile of operable pixels (Req. $=95 \%)$ for science. Non-operability can be either a permanent or a transient feature. In the category of permanently inoperable pixels for science we intend to consider:

- pixels disconnected from the MUX (mainly with a missing bump),

- pixels with Random Telegraph Signal (typical pixel with traps under the oxide of the gate of the pixel source follower),

- pixels not responding to light due to low quantum efficiency in each band of observation,

- pixels responding weirdly (pixel response to illumination which cannot be corrected by the non-linearity lookup table),

- hot pixels (defined for a given threshold on the master dark),

- pixels showing a baseline out of the useful range.

In the transient category of inoperable pixels we notice: 
- the pixels showing a ramp with a primary or a secondary particle interaction within the sensitive substrate,

- the pixels identified as producing too high background signal originating from de-trapping,

- the pixels with a very high capture probability of charge carriers.

Characterization products Table 1 summarizes the expected products of the detector chain calibration pipeline which should be applied on ground characterization data.

Table 1. NISP products: expected pixel maps produced by the detector chain calibration pipeline which will reduce data taken during the Flight Runs of the Euclid Consortium ground characterization campaign

\begin{tabular}{|l|l|l|}
\hline Product & Description & Destination \\
\hline master darks & dark in the science readout mode & SGS-NISP \\
\hline master noise & slope noise in the science readout mode & SGS-NISP \\
\hline nl LUT & non-linearity correction LUT (input: flux) & SGS-NISP \\
\hline la LUT & latency LUT for masking (input: previous flux) & SGS-NISP \\
\hline flat LUT & flat fielding LUT & SGS-NISP \\
\hline non-operable & mask of permanent non-operable pixels (all bads) & SGS-NISP \\
\hline ipc & Inter Pixel Capacitance with Single Pixel Reset & SGS-NISP \\
\hline cg & conversion gain (cnt per e-) & SGS-NISP \\
\hline baseline & mean and STD of the first group after Reset & NISP \\
\hline dpix & disconnected pixels & NISP \\
\hline bad baseline & pixel with too high or too low baseline & NISP \\
\hline bad response & non-responding or badly responding pixels & NISP \\
\hline rts & pixels with Random Telegraph Signal & NISP \\
\hline trapping / de-trapping & trapping coefficients (model) & NISP \\
\hline
\end{tabular}

\subsection{NISP detector chain overview}

This work is mainly focused on the most challenging contribution to the pixel calibration of the pixel response, i.e. the sensitivity of the non-linearity correction to the trapping/de-trapping effects.

Two additional Euclid papers on the conversion gain measurement per pixel and the pixels with Random Telegraph Signal are presented for this conference $\left(\operatorname{see}^{4} \mathrm{and}^{5}\right)$.

To characterize and correct the non-linearity of the pixel response it is important to underline different contributions of the transfer functions establishing the relation between the number of impinging NIR photons and the number of Analog to Digital Converter units (ADCu) or counts (cnt).

Pixel response calibration strategy The main idea driving the calibration strategy requires a method able to separate the measurement of the contribution of the transfer function in the charge domain and the electronic transfer function in the voltage domain. This hypothesis can be simplified by assessing that it is necessary to correct first the pixel response by all transfer function in the voltage domain before deriving any 'varactor' effect of the pn-junction (quadratic in first approximation) and then to study the trapping/de-trapping contributions in the charge carrier domain.

Our hypothesis is sustained by the repeated observation that a direct polynomial fit (orthogonal or not) of the pixel response obtained from 20 ramps under a stable illumination (better than 1\%) are dependent of the traps 
states (free or occupied) and consequently dependent of the illumination history (dark, zodiacal background of $2 \mathrm{e} / \mathrm{s}$ or same flux). A typical standard deviation of the linear part of the fit (the expected true flux to be used to generate the correction lookup tables) obtained with a 4 th order orthogonal polynomial fit is around $10 \%$ in a same pixel over 20 ramps of the same illumination within 1\% (the stability is controlled by a cold photo-diode in the focal plan). Surprisingly the standard deviation over the ramps is too high compared with the expected standard deviation from the statistics and the readout noise although the residuals of the fit per ramp are good. This point will be discussed in section 4 .

Detector chain scheme and transfer function of the components Figure 2 shows a simplified view of the main components contributing to the different effects responsible to the inaccuracy of the detector chain response calibration. Figure 3 summarizes in a more synthetic view the matching between the physical components and

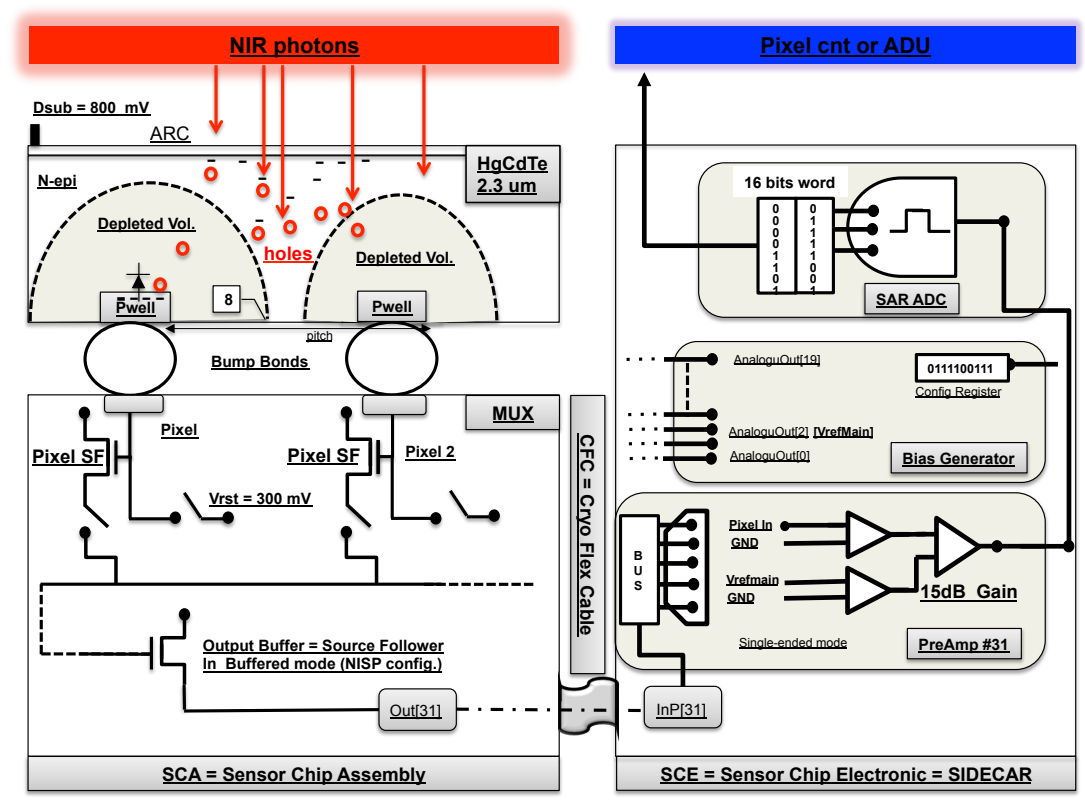

Figure 2. Simplified schematics of the main components of the detector chain. All these components impact the response of the $\mathrm{HgCdTe}$ detector chain.

the transfer function of the signal.

The main components of the SIDECAR ASIC used for NISP readout are the 33 preamplifiers: 0 to 31 for the 32 channels of science and reference pixels and one channel to sample the feedback of the reference voltages. Data from channel 33 are called telemetry in what follows.

The main components of the Multiplexer bonded with the HgCdTe are the output buffers (one source follower per channel) and the source followers of the pixels (SF). Various components and effects are considered for the HgCdTe sensitive volume. The transfer function corresponding to the pn-junction (p-well to collect holes) is the charge to voltage conversion factor ( $\mathrm{CVF}$ in $\mu \mathrm{V} / \mathrm{e}-$, also named the transimpedance gain). The sensitive volume, N-epi layer is responsible for two transfer functions which are the quantum efficiency (QE) and the charge collection efficiency (CCE). From an experimental point of view we are measuring the product of the two transfer functions. The first one is related to the ratio between the photo-generated carriers and the incoming photons. The second refers to the collection efficiency of the carriers by the depleted volume. This is a dynamical behaviour because of the relation between the density of free traps and the extension of the depletion volume during the integration time. This relation is also dynamical in the sense that the densities of traps susceptible to collect charge carriers and occupied traps susceptible to release charge carriers depend on the illumination history of the pixel. 


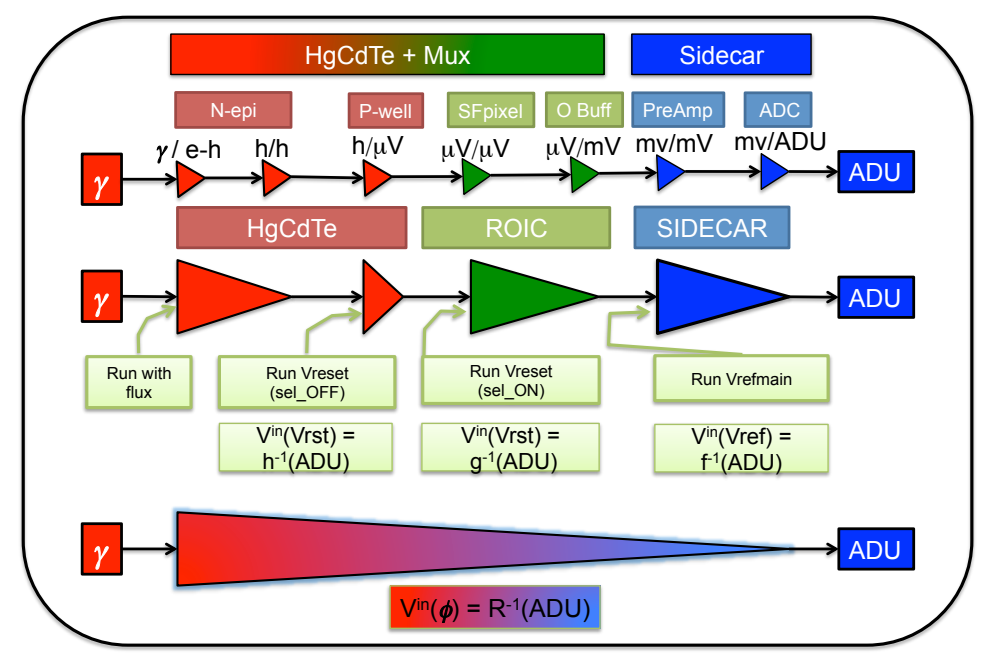

Figure 3. Scheme of the main transfer functions of the pixel response corresponding to the main components of the readout chain and its electronics of Figure 2.

Figure 4 shows the flat field computed from two independent measurements: the quantum efficiency maps provided by the acceptance test of NASA and the Least Square Fit of the signal of the ramp for a stable and flat illumination around $90 \mathrm{e} / \mathrm{s}$. The flat is normalized by the quantum efficiency map after dark subtraction to generate the illumination flat field image. This image should have a uniformity better than $1 \%$ on the full array since we have checked that the flatness of the illumination is better than 1\% RMS. The right part of Figure 4 shows the residual dark after an illumination ramp. The two images are fairly correlated (same trapping and detrapping areas). The average flatness value in the 'smiley' shape is lower. This is interpreted as a lower QE (more precisely lower CCE) for this illumination after a dark acquisition than the QE taken from the NASA maps. It is not surprising we notice that the QE maps are measured with strong fluxes without darks interleaved. On the contrary the ramp used to compute the LSF of this flat field image follows a dark exposure. Then in this case the number of free traps is clearly higher. This is in favour of the need for a better control of trapping/de-trapping contribution to the signal measurement.

Different contributions to the non-linearity from the components In Figure 3 we see the transfer functions of the pixel response through the three components: the $\mathrm{HgCdTe}$, the ROIC and the SIDECAR ASIC. To disentangle the non-linearity contributions, the circuitry configurations offer various possibilities to apply reference voltage at different stages of the detector chain. The different inputs are reported in Figure 3. Two of them are dedicated to verification (vrefmain, vreset with the reset switched off or open state) whereas the reset voltage on the gate of the source follower (SF) keeping the reset selection switched on is used to generate the electrical transfer function $\left(g^{-1}(A D C u)=V\right.$ of Figure 3$)$ which includes all voltage contributions down to the digital conversion of the ADC.

\section{DETECTOR CHAIN CHARACTERIZATION}

\subsection{Test flow overview}

\subsubsection{Definitions}

The test flow of the ground characterization performed by the NISP detector team (from IN2P3*) has been specified through a dedicated terminology. This dictionary has been introduced to generate a synthetic and general description to script all possible runs used for the detector chain characterization.

\footnotetext{
${ }^{*}$ National Institute of Nuclear and Particle Physics
} 

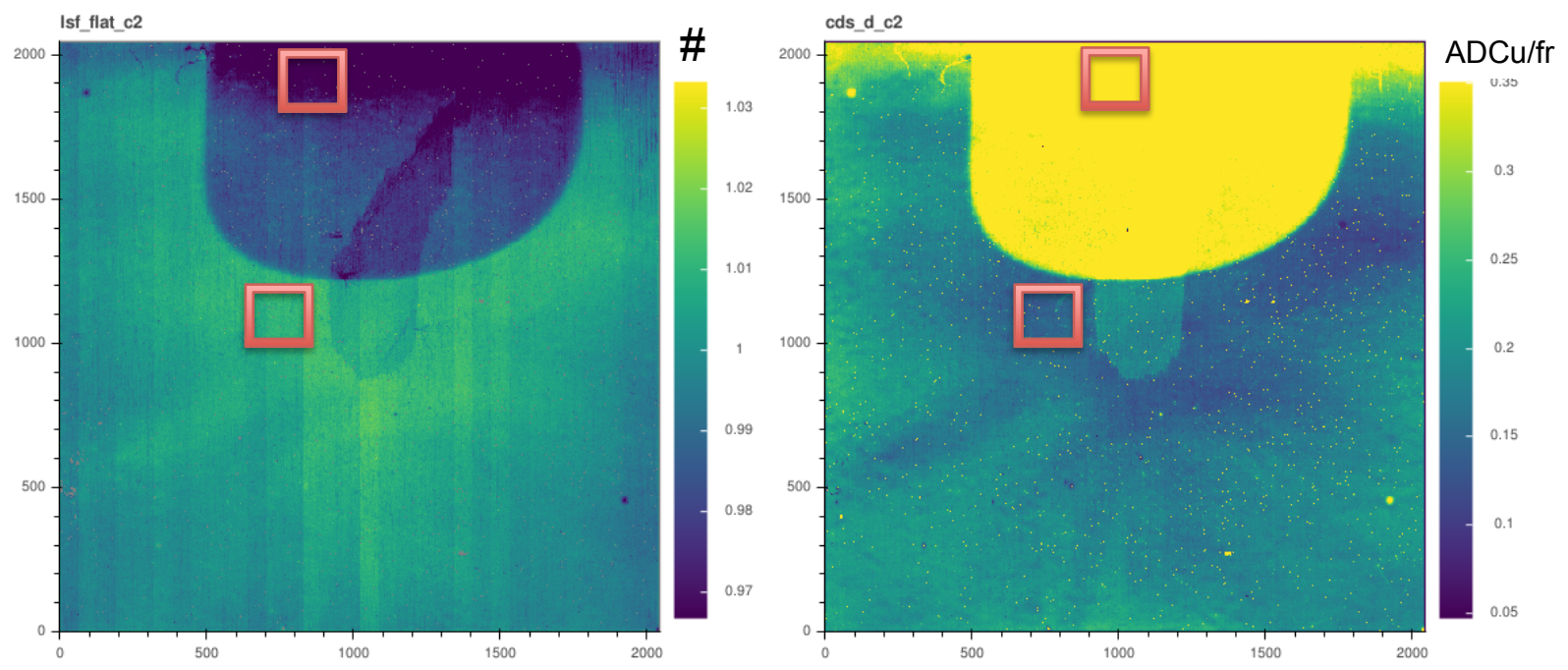

Figure 4. Left: Flat Field computed from a stable flat illumination after dark subtraction and normalization by a QE maps obtained from the NASA acceptance test. The illumination follows a dark ramp. Right: image of the residual dark (right) after illumination. These images are taken from the data QC of SCA 18268 of Flight Run 2 on Pegasus.

Figure 5 presents the generic description of encapsulation starting from the most general which is a run to the smallest building block: the exposure with a single ASIC configuration, one readout mode and one environment status. The pseudo-code of specification has been used to generate the a scheduler of the test flow covering the 45 days of the test flow. The fully automatized asynchronous data acquisition system (DAS) controls and

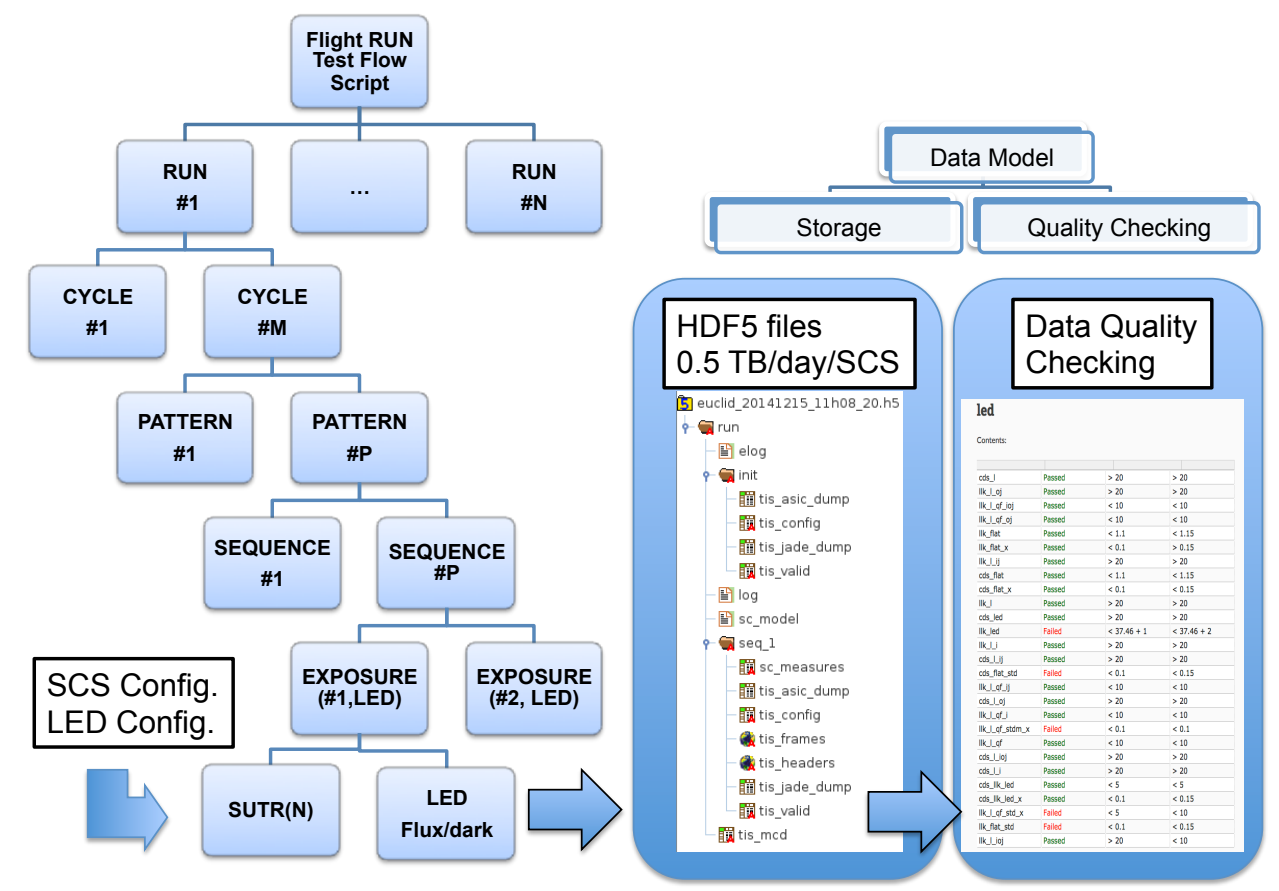

Figure 5. Scheme of a generic run of the test flow. This description dedicated to NISP allows to specify all possible runs of the ground characterization campaign in a unique and general way which is translated into a pseudo-code for the scheduler of the DAS. 
monitors the illuminations when it executes the list of the specified runs with dedicated detector configurations. At the end of each run the DAS generates a report on data quality checking (QC) based on pixel maps with systematic comparison between current and reference maps (absolute or relative). The Data QC functions produces few hundreds of histograms and pixel maps per run to validate the expectations from the scheduler point of view. The analyses are performed per pixel but also with spatial averages over rows, columns, channels and arrays to highlight some possible electronics features or instabilities. In summary, the DAS developed for the Euclid ground testing campaign can be seen as a scheduler of asynchronous distributed object or distributed functions over a network to produce a huge amount of Detector Data and to control and assess their quality.

\subsubsection{Runs of the test flow}

When the test campaign started in 2017, the main programmatic requirement was on the maximum duration (50 days) allocated to the characterization per SCS. The test flow has been derived to match this time scale keeping the objective of $1 \%$ accuracy on the corrected pixel response. A list of runs presented in Table 2 has been established to cover as much as possible different illumination scenarios to generate the largest data set with trapping and de-trapping effects.

Table 2. List of the runs used during the test flow. Most of them are repeated at the three nominal operating temperature $\mathrm{OT}=90 \mathrm{~K}, 85 \mathrm{~K}, 80 \mathrm{~K}$

\begin{tabular}{|c|c|c|c|c|}
\hline $\begin{array}{l}\text { Run type } \\
\text { name }\end{array}$ & $\begin{array}{l}\text { Flux cfg } \\
(\mathrm{e}-/ \mathrm{s})\end{array}$ & $\begin{array}{l}\text { Pattern } \\
\text { nb. ramps }\end{array}$ & $\begin{array}{l}\text { \# Iter. } \\
\text { (stat 1\%) }\end{array}$ & calib. purpose \\
\hline \multicolumn{5}{|l|}{ Reset runs } \\
\hline Baseline & 0 & 16 & 1000 & First Gr. + kTC \\
\hline Vrst & 0 & 3 & $64 / \mathrm{Vrst}$ & non linearity \\
\hline SPR & 0 & SPR mode & $16 /$ Vrst & IPC per pixel \\
\hline \multicolumn{5}{|l|}{ Dark runs } \\
\hline Dark sci. & 0 & 500 & 50 & Master dark \\
\hline Dark long & 0 & 5000 & 4 & RTS + ref. dark \\
\hline \multicolumn{5}{|l|}{ Flat runs } \\
\hline Flux zodiacal bkg & 2 & 400 & 60 & non-linearity + latency short \\
\hline Flux & $8,16,28,52,90$ & 400 & 30 to 5 & non-linearity + latency short \\
\hline Flux + short dark & $4,8,16,28,40,52$ & $400+800$ & 30 to 5 & non-linearity + latency short \\
\hline- & $60,80,90,100,120$ & $400+800$ & 20 & non-linearity + latency short \\
\hline- & $140,160,200,280$ & $400+800)$ & 20 to 6 & non-linearity + latency short \\
\hline High Flux + short dark & $400,600,800$ & $100+1200$ & 6 & non-linearity + latency short \\
\hline- & $1000,1200,1400$ & $100+1200$ & 6 & latency short \\
\hline Flux + zodiacal bkg & $8,16,28,52,90$ & $400+800$ & 20 & non-linearity + latency short \\
\hline Flux + long dark & $4,8,16,28,40$ & $400+5000$ & 5 & non-linearity + latency long \\
\hline- & $52,60,80,90,160$ & $400+5000$ & 5 & non-linearity + latency long \\
\hline- & $1200,2000,4000$ & $100+5000$ & 3 & latency long \\
\hline Mono-chromator & $50-15 \lambda \mathrm{s}$ & $400-100$ & 5 & spectral response \\
\hline
\end{tabular}




\subsection{Summary of the Flight Runs 2017-2018}

The CPPM detector characterization laboratory developed 2 test facilities dedicated to the Euclid NISP ground characterization campaign. Each facility is able to characterize two detectors in parallel with the same illumination sequences and the same focal plan temperature. Therefore a Flight Run is composed of 45 days of tests with 4 SCS in parallel. We kept the naming convention used during the test campaign i.e. FR2 to FR5. It is not the purpose of this work to describe in details the characterization facilities. Nevertheless, to highlight the success of the test campaign and the effectiveness of the characterization facility in terms of hardware, software and human resources, the Figure 6 represents the yield of the test flow. Yield is derived from the number of frames effectively stored on disk as function of time. The dotted grey line represents the ideal acquisition campaign corresponding to a increase of the number of frames stored on disk if a continuous readout of frames is sustained during the full duration of the Flight Run campaign. Then the yield is the ratio between the true number of frames and the ideal one for a period standing from the first cold run to the last cold run of the scheduler. Figure 6 illustrates the complexity of the test flow by showing on the left and in background colors the operating temperatures and on the right the illumination status versus time.
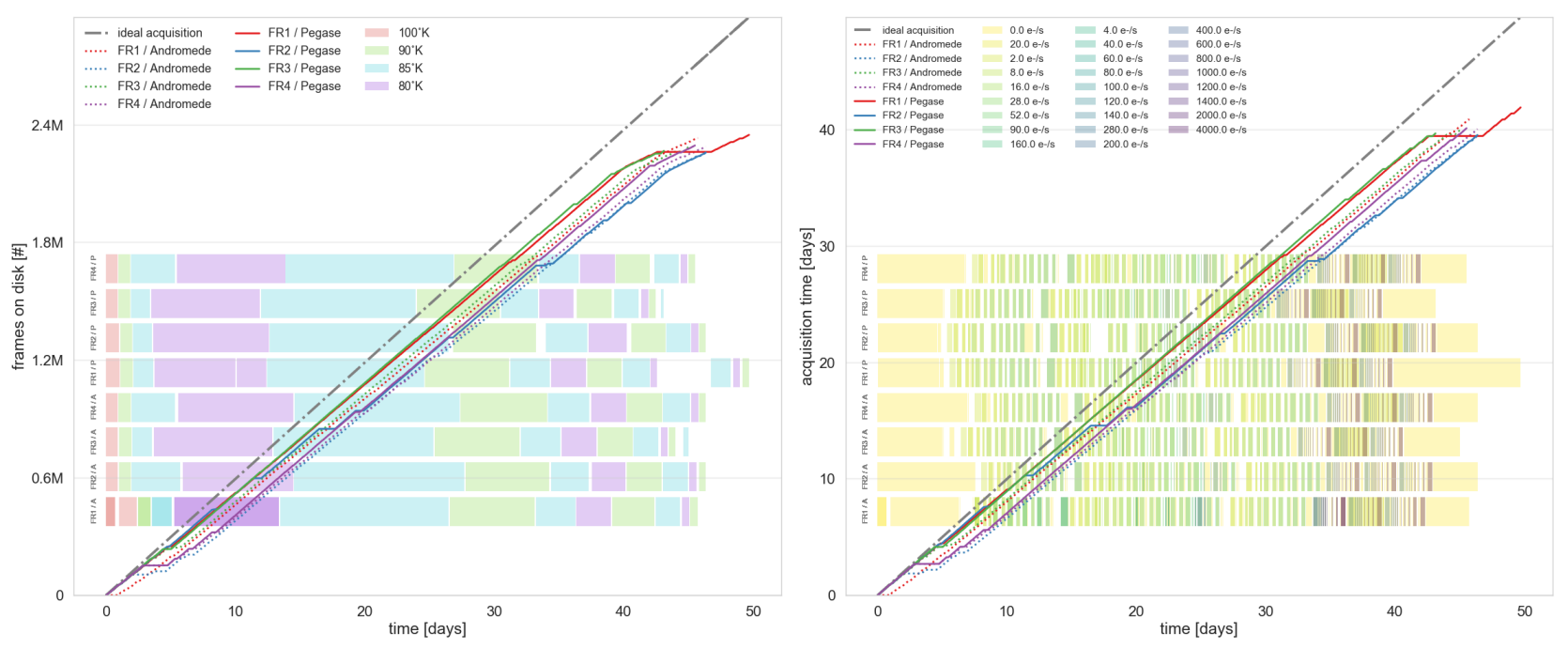

Figure 6. Figures of Merits of the FR2 to FR5 characterization yields computed from the frames stored on disk (left) and the acquisition time (right). The ideal characterization corresponds to the straight line versus time standing for a continuous acquisition during more than 45 days devoted to the flight Runs. The color bands in the background shows the operating temperature of the SCAs (left) and the flux status of the illumination set up during the test flow (right). These measurement shows the complexity of the test flow. Pegasus and Andromeda are the names of the 2 cryostat of the CPPM test facility.

The main results illustrated in Figure 6 are reported in Table 3. An extremely high yield of $80 \%$ has been sustained during more that $4 \times 50$ days of testing, thanks to the huge human effort and to a well designed automation. The main delays observed during the test campaign are mainly due to general power blackout in the Marseille area due to storms and one important Pulse Tube failure. The second reason of delay was that tests with the mono-chromator (one for both) and the operating temperature changes (OTs $=100 \mathrm{~K}, 90 \mathrm{~K}, 85 \mathrm{~K}$, $80 \mathrm{~K}$ ) have not been automatized (see Figure 6 left for the temperature time lines per Flight Run). The data QC processing is automatically performed to report and to validate the run. This validation is based on a summary through tables with fail and warning flags for each variables computed and compared with reference values extracted from the history of the test flow. A screen shot of the data QC processing is shown in Figure 5 (right). 
Table 3. Summary table of the 4 Flight Runs with SCS-Id, date, duration and the two Figures of Merit of the Euclid ground testing campaign of the Flight SCAs.

\begin{tabular}{|l|l|l|l|l|l|l|l|l|l|}
\hline $\begin{array}{l}\text { FR } \\
\#\end{array}$ & $\begin{array}{l}\text { Cryo } \\
\text { And. } \\
\text { Peg. }\end{array}$ & $\begin{array}{l}\text { SCS Id } \\
18 \#-18 \#\end{array}$ & $\begin{array}{l}\text { Beg. time } \\
\text { date } \\
\text { h.mm:ss }\end{array}$ & $\begin{array}{l}\text { Duration } \\
\text { days } \\
\text { hh:mm:ss }\end{array}$ & $\begin{array}{l}\text { Runs } \\
\#\end{array}$ & $\begin{array}{l}\text { Ramps } \\
\#\end{array}$ & $\begin{array}{l}\text { Frames } \\
\#\end{array}$ & $\begin{array}{l}\text { FoM } \\
\text { acq. } \\
\mathbf{\%}\end{array}$ & $\begin{array}{l}\text { FoM } \\
\text { frames } \\
\mathbf{\%}\end{array}$ \\
\hline 2 & A & $249-278$ & $\begin{array}{l}2017-09-07 \\
14: 51: 49\end{array}$ & $\begin{array}{l}45 \\
18: 48: 23\end{array}$ & 157 & 38683 & 2335668 & $\mathbf{8 9 . 4}$ & $\mathbf{8 5 . 9}$ \\
\hline 2 & P & $268-284$ & $\begin{array}{l}2017-09-07 \\
14: 08: 43\end{array}$ & $\begin{array}{l}50 \\
17: 38: 57\end{array}$ & 159 & 61840 & 2359329 & $\mathbf{8 3 . 0}$ & $\mathbf{7 8 . 3}$ \\
\hline 3 & A & $269-452$ & $\begin{array}{l}2017-11-15 \\
13: 42: 45\end{array}$ & $\begin{array}{l}46 \\
15: 05: 09\end{array}$ & 152 & 38582 & 2262438 & $\mathbf{8 4 . 9}$ & $\mathbf{8 1 . 7}$ \\
\hline 3 & P & $453-632$ & $\begin{array}{l}2017-11-02 \\
14: 30: 44\end{array}$ & $\begin{array}{l}46 \\
12: 51: 39\end{array}$ & 147 & 38560 & 2265078 & $\mathbf{8 5 . 2}$ & $\mathbf{8 2 . 0}$ \\
\hline 4 & A & $280-282$ & $\begin{array}{l}2018-01-13 \\
19: 00: 17\end{array}$ & $\begin{array}{l}45 \\
2: 39: 13\end{array}$ & 149 & 38818 & 2292593 & $\mathbf{8 8 . 8}$ & $\mathbf{8 5 . 6}$ \\
\hline 4 & P & $285-548$ & $\begin{array}{l}2018-01-14 \\
21: 01: 33\end{array}$ & $\begin{array}{l}43 \\
4: 52: 00\end{array}$ & 143 & 38619 & 2273113 & $\mathbf{9 2 . 1}$ & $\mathbf{8 8 . 6}$ \\
\hline 5 & A & $221-267$ & $\begin{array}{l}2018-03-06 \\
14: 59: 51\end{array}$ & $\begin{array}{l}46 \\
10: 32: 18\end{array}$ & 146 & 39573 & 2295328 & $\mathbf{8 6 . 5}$ & $\mathbf{8 3 . 2}$ \\
\hline 5 & P & $272-458$ & $\begin{array}{l}2018-03-06 \\
19: 22: 40\end{array}$ & $\begin{array}{l}45 \\
14: 35: 49\end{array}$ & 150 & 38634 & 2300413 & $\mathbf{8 8 . 2}$ & $\mathbf{8 4 . 9}$ \\
\hline
\end{tabular}

\section{DETECTOR CHAIN CALIBRATION}

\subsection{Methods}

\subsubsection{Rationale for a pixel response calibration pipeline}

The rationale for the pixel calibration flows down from the requirements mentioned in section 2.

Readout mode and SCE configuration The data bandwidth sustained by the Data Acquisition Software enables a full readout and storage of all frames of the ramps for the 2 SCS in parallel. Therefore, sampling up the ramp was systematically used for the readout mode. It has been checked during the Pilot Runs that there is no difference in the noise properties between data taken with a MACC mode implemented inside the SIDECAR which not transmit the frames between the groups and data obtained in SUTR and computed off-line in the same MACC mode. This is not surprising since all frames are sampled by the ADCs even if the MACC mode is selected by the Euclid flight firmware loaded in the SIDECAR. A $15 \mathrm{~dB}$ value of gain has been chosen for the preamplifiers with a single ended bus configuration. A bias of $500 \mathrm{mV}$ has been set between Dsub substrate voltage and the reset voltage of the P-well. With a $100 \mathrm{kHz}$ pixel clock, the full frame $(2048 \times 2048)$ is read out in $1.4548 \mathrm{~s}$ with 32 channels in parallel.

Baseline The in-flight information about the flux (See Ref. ${ }^{6}$ for the definition of the slope computation and the associated quality factor (QF) of the fit used in NISP) transmitted to the ground is constrained by telemetry/day budget. None of the group $\mathrm{ADCu}$ values is transmitted to the ground in science observation modes. It is then of relevant interest to have a determination of the baseline after reset and its scatter from ground tests (see Table 2 for details on this run). Possible reset anomalies are well traceable by comparing the mean value of the 
first frame to the mean value of the first group. The first two CDS are also compared. This noise has to be well controlled since it could contribute to the systematic error when a non-linearity correction is applied to the measured slope. Thanks to the large number of iterations (1000), the precision of this measurement allows to be well under the Reset noise per ramp.

Darks Darks acquisitions are of two types: short and long darks (see Table 2). Short darks with SUTR(500) are typically repeated 62 times for each operating temperatures $(80 \mathrm{~K}, 85 \mathrm{~K}, 90 \mathrm{~K}, 100 \mathrm{~K})$ to derive for each science MACC readout mode the master dark and the master noise of the slope after reference pixel subtraction. A larger value of 500 frames instead of the nominal 400 has been chosen in case of a necessity to increase the integration time for science observation during the mission. Reference from ground data will be available in this case.

Zodiacal background illumination Zodiacal background which is expected to be around 2 e-/s in-flight is of major importance to understand the steady states of the pixels since they will "see" in average such flux during at least 24 hours without higher illumination from stars or galaxies. A cycle with 60 iterations has been programmed at each OTs to produce a zodiacal reference data sample.

Electrical transfer function for non-linearity correction We have mentioned in section 2 that in order to build the non-linearity correction lookup tables for each readout mode it is necessary to measure the true linear flux with a systematic error lower than $1 \%$. The methodology foreseen for NISP is defined in Ref. ${ }^{7}$ This method uses a polynomial fit (orthogonal or not) up to the 4 th degree. This should give enough accuracy on the "true" linear flux. As mentioned previously, the standard deviation of the true flux is observed to be for some type of acquisitions and for some groups of pixels twice higher than the expected Poisson and readout noise contributions. After many tests and analyses in a broad range of fluxes and various illumination histories (illumination preceded by a dark, a zodiacal background and an equivalent flux) we observed that it could become problematic to find a unique set of correction coefficients although the residuals of the fit were good and not structured along the ramp.

This observation leads us to try to disentangle different contributions to the non-linearity of the pixel response $(\mathrm{V} / \mathrm{V}, \mathrm{e} / \mathrm{V}$ or trapping) in order to isolate the trapping and de-trapping contributions. We hope to be able to compute the "true" linear flux required by the non-linearity lookup table correction method with a better control of the systematic errors.

A second argument in favour of the (SF) transfer function method is that we will be able to verify the nonlinear behaviour of the pixel response with a pretty good statistical accuracy that is unreachable with low flux illumination due to the number of ramps required by the averaging (thousands of ramps).

A third argument for this method is that the baseline reset method can be executed in-flight and then could be useful for in-flight calibration or verification. This could be a complementary method to flat field obtained with the calibration unit.

The corresponding electrical runs dedicated to this measurement are identified as reset voltage runs since the electric transfer function is constructed by changing the reset voltage on different input stages of the readout chain. The different possibilities used for the flight Runs are reported in Figure 3.

Non linearity correction and trapping effects The second objective is to develop a trapping and detrapping model either to mask the transient inoperable pixels or to correct the illumination. Indeed we expect by working on different states of occupied traps preceding the illumination, to develop a trapping and de-trapping model which will be able to fit as much a ramp of illumination with de-trapping and trapping of charge carriers as a dark or zodiacal ramp with de-trapping. Thanks to the data acquisition system which time stamps all event and frames all along the 45 days of acquisition, we expect to be able to constraint with many different illumination scenarios the parameters of the trapping and de-trapping model with a global fitting procedure. 
For that purpose we define different scenarios repeated many times at many fluxes and at the 3 operating temperatures. The targeted fluxes are mainly those expected during the in-flight calibration with the NISP Calibration Unit (NI-CU fluxes are 28, 52, 90, 160, $280 \mathrm{e}-/ \mathrm{s}$ ) but lower fluxes are also tested with 30 iterations to reduce the relative precision below $1 \%$ per pixel. The illumination scenarios are described by the simple notation: (flux + short dark), (flux+long dark), (flux+zodiacal background), (flux+flux). See Table 2 for a detailed description of the non-linearity runs.

De-trapping effects (fast and slow components) The de-trapping model, that will be compatible with the trapping effects, will be based on two types of illumination patterns (see Figure 5 for the definition of a pattern). The ones with long dark $(7274 \mathrm{~s})$ will be used mainly to measure the slow decay time constant and the ones with short darks (1163s) reproduced with a higher statistics. These runs, reported in Table 2, will be first used to fit the density of occupied traps versus the preceding illumination and the fast decay time constant.

De-trapping after saturation of the P-well High flux saturating the P-well of the diode (120 ke- with a Bias of $0.5 \mathrm{~V}$ ) are also executed with short and long darks (See Table 2) to measure the increase of trapping and de-trapping due surface states appearing as active due to disappearance of the collecting electric field profile or in order words the shrinking of the depletion volume.

Inter pixel capacitance (IPC) with single pixel reset method (SPR) The single pixel reset has been implemented into the Euclid flight firmware of the SIDECAR. With 64 different register configurations of the single pixel Grid the full array is covered in a short time (few hours). Then a per pixel IPC map is produced thanks to the DAS. Three runs with three different biases values $(0.35 \mathrm{~V}, 0.5 \mathrm{~V}, 0.625 \mathrm{~V})$ have been produced to quantify the IPC sensitivity to the voltage difference between the two resets.

Temperature Sensitivity The in-flight passive cooling of the NISP focal plane does not allow to proceed with a ground testing at a single temperature since the OT is not predictable. Three operating temperatures have been chosen to cover the range of expected OTs with margins. The parameters used to correct the response function will be compared for the three OTs. To stay within the 45 days of testing per SCS, OT=80K and OT=90K cover fewer scenarios than $\mathrm{OT}=85 \mathrm{~K}$ keeping at least the same statistics used for the nominal illumination fluxes of the NI-CU that will be available in-flight (20 iterations for each pattern and each nominal flux).

\subsection{Preliminary Results from the Flight Runs}

A systematic reporting on the 4 Flight Runs is out of the scope of this conference paper. We focus here on results which underline the pixel calibration strategy and show the tricky part of the pipeline.

\subsubsection{V/V non-linearity correction}

From pixel Source Follower to the ADC units A specific run dedicated to the V/V measurement has been applied (see Reset Sel ON in Figure 3). The pixel reset switch is represented in Figure 2. Using Telemetry measurement of the applied Reset voltage, it is straightforward to construct the pixel response function in Volt with a step of one LSB in the configuration bit word $(\sim 2 \mathrm{mV} / \mathrm{LSB})$.

It is nevertheless of prime importance to verified the linearity of the voltage applied in the range $0.3-0.8 \mathrm{~V}$ before fitting the correction function. Both the $-3 \mathrm{~dB}$ gain set on the telemetry preamplifier and the setting value of the vrefmain voltage reference put the $\mathrm{ADCu}$ values in a small, central and linear range of the ADC.

To validate the linearity of the telemetry versus voltage, we have studied with a linear fit the telemetry value $(\mathrm{ADCu})$ versus the configuration voltage or equivalently the configuration bit word. Figure 7 (left) shows such verification on the reset voltage linearity. The interesting feature, raised from this figure of residuals, is the appearance of periodical voltage steps as function of the configuration value.

One way to stress this feature is to plot the consecutive difference of telemetry values versus the voltage steps called CDS in what follows. The right part of Figure 7 shows the CDS values of the telemetry versus 

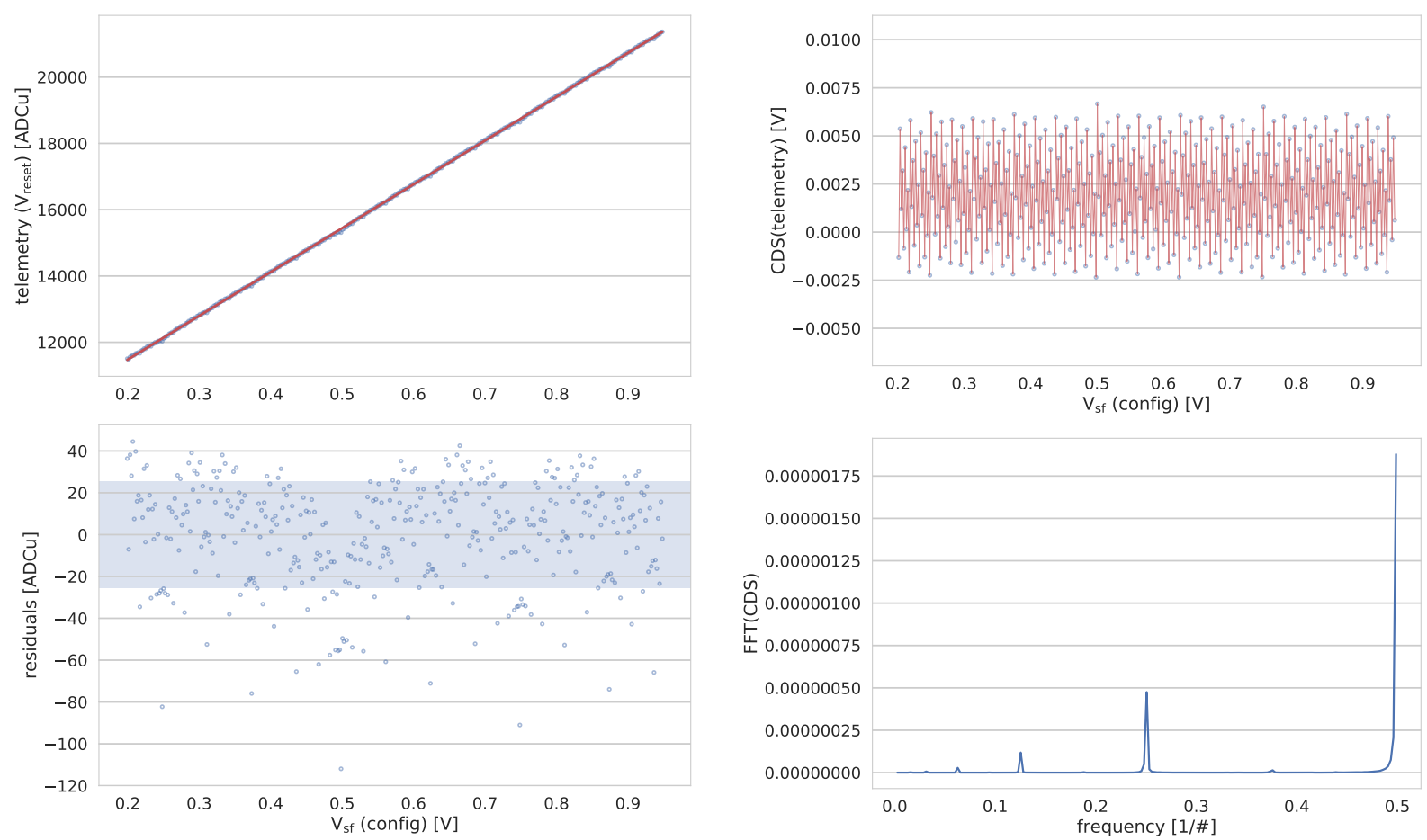

Figure 7. Left: Linearity of the Reset Voltage applied during electrical test on the Gate of the Source Follower with on state. Right: vreset difference of two consecutive voltage with one LSB flip (top) and the corresponding spectrum of the Fourier Transform (bottom). We clearly stress a bit flip effect of the DAC with the frequencies $\frac{1}{2^{n}}$ with $n=1,2,3, \ldots$

the configuration voltage. The periodicity is obvious. To understand the reason of such periodicity a Fourier Transform as a function of the bit word translated into an integer word has been computed. The Fourier spectrum shows amplitudes corresponding to the frequencies belonging into the series $\frac{1}{2^{n}}$ with $n=1,2,3, \ldots$. We conclude that this effect is clearly a consequence of the bit flips of the digital to analog conversion (DAC) component. Therefore the inverse transfer function has been applied to the telemetry values and not on the configuration voltage. The linear fit over more than 350 values of reference voltage gives us the exact relation between voltage and telemetry ADCu.

Once the linearity of the input voltages is verified the transfer function response is measured over the full ADC range with all necessary bit words of the DAC and measuring the baseline of the pixel over a number of ramps and a number of frames per ramp sufficient to minimize the errors due to the readout noise and the kTC noise. The results are presented in Figures 8 and 9 where, for each degree of the polynomial function, the global residual of the fit and the zoom in the useful range for science (from the baseline of the pixel to $63000 \mathrm{ADCu}$ ) are plotted. We clearly see that the 4 th degree is required to decrease the residuals below $0.1 \%$. Next, we generated the $\mathrm{ADCu}$ to the source follower input voltage inversion function for the 4th degree polynomial. A systematic study on the cost and of the benefit of using of a higher polynomial order to gain one or two thousands of ADCu in the ADC saturation part will be performed. We are not fully convinced that the benefit from the increase of the dynamical range justifies the risk of a strong increase of the systematics after applying this correction.

\subsection{2 e/V non-linearity correction}

Applying the inverse transfer function translating $\mathrm{ADCu}$ into voltage on the gate of the pixel SF gives access to the e/V transfer function. Under a strong illumination, covering the 16 bits ADCu range in typical spectroscopic ramp of 400 frames (565s), we expect to observe a typical varactor effect of a pn-junction. ${ }^{8}$ Nevertheless, we notice that trapping and de-trapping contributions are dominant during the first 100 frames. 

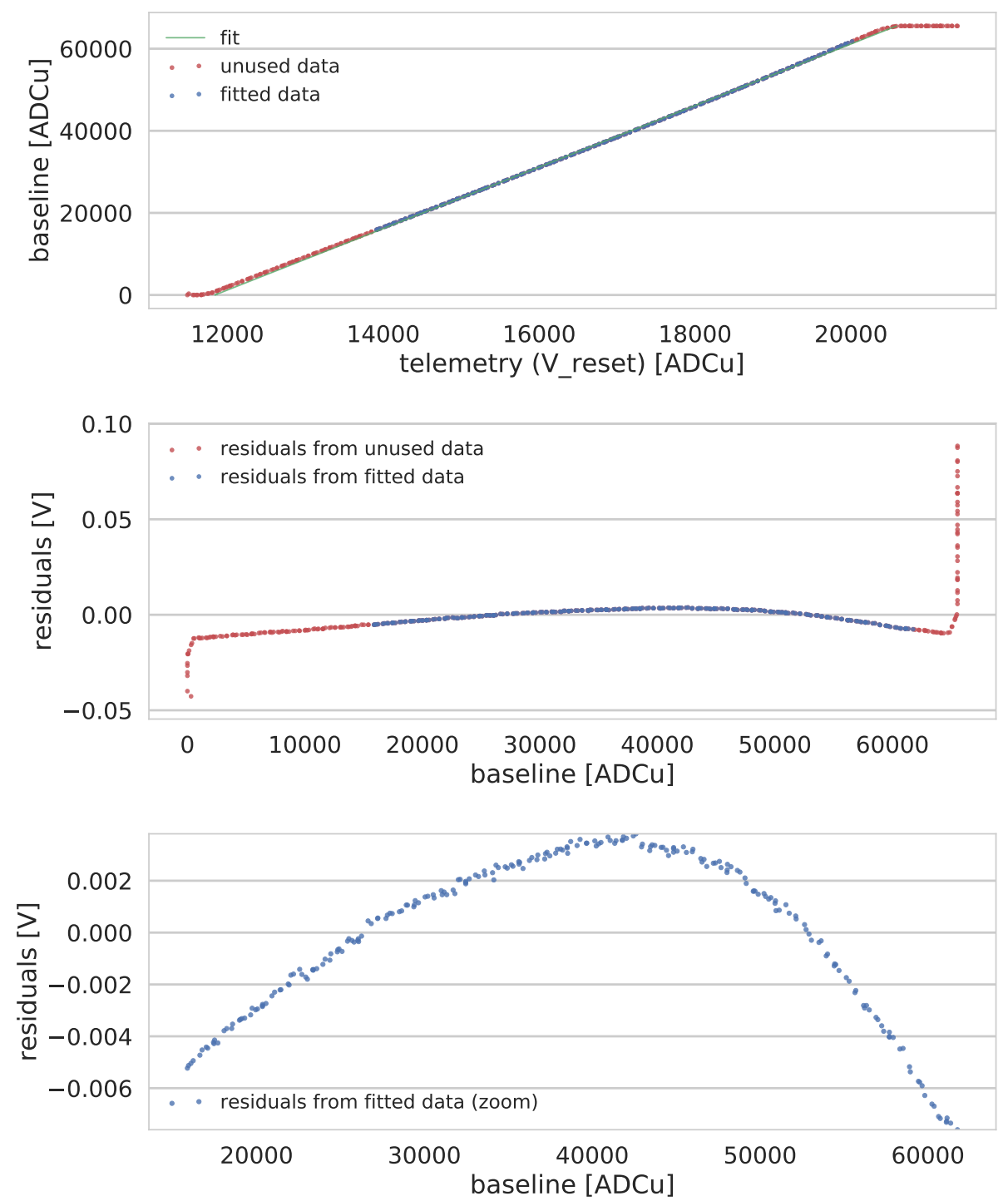

Figure 8. Voltage response to Vreset SEL ON run of Pixel $(2000,1036)$ of SCS 18628 . The residuals to the fit with a 1 th order polynomial is shown (bottom).

To verify the e/V contribution to the pixel non-linearity we show in Figure 10 the residual to a linear fit of the pixel response for two groups of 64 pixels after and before the application of the SF inverse function presented in section 4.2.1. The two groups are reported in Figure 4. They are representative of two different populations with a strong and weak latency.

Figure 10 is very instructive if we compare the panels on the left with those on the right as they correspond to the after (left) and before (right) the application of the SF correction, respectively. The top and bottom panels correspond to two different groups of pixels (see squares in Figure 4) showing a difference in the measured latency of the order of two (higher persistence observed for the group of pixels corresponds to the residuals at the bottom of Figure 9). Important comments from this figure can be derived.

- The residuals without correction of electrical response (just a translation of $\mathrm{ADCu}$ into volt through a constant transimpedance gain and conversion gain ratio to keep the same units), the two plots on the right 

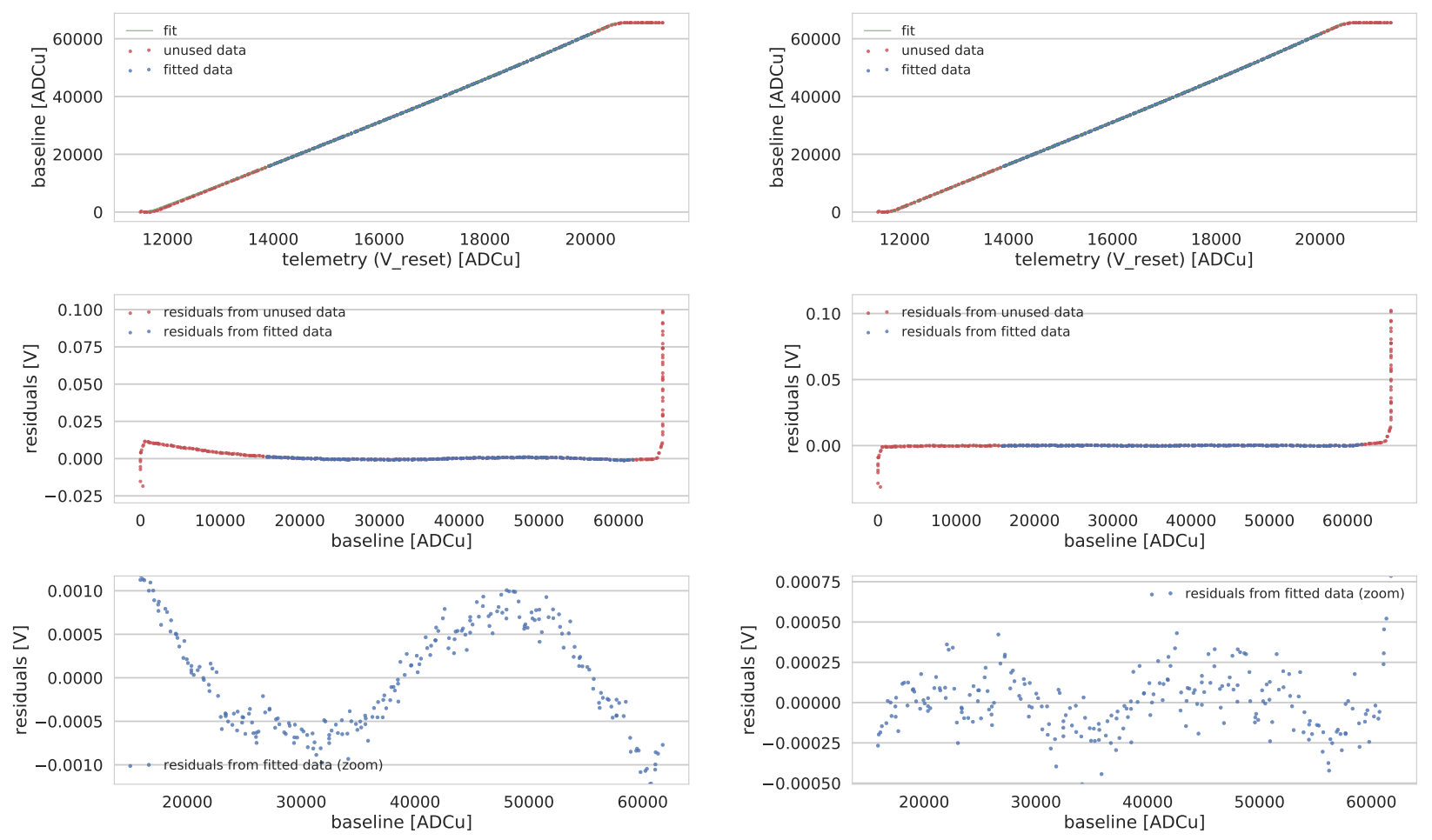

Figure 9. Voltage response to to the reset run of Pixel $(2000,1036)$ for SCS 18628 . The residual of the fit with a 2 nd order polynomial is shown in bottom left. The residual of the fit with a 4 th order polynomial is shown in bottom right.

of Figure 10, show very different behaviours depending on the trap density. This confirms the fact that for some pixels a direct fit of the pixel under constant illumination with a 4th order polynomial will not be stable for different illumination history. This can explain the $10 \%$ RMS of the linear part of the fit observed on a full array.

- The high-end of the residuals for the non corrected data presents a broader dispersion among pixels. On the contrary, the corrected residuals present a very sharp dispersion. This is the proof of the effectiveness of the SF pixel correction and it shows that we face the non linearity of the pn-junction. It suggests a very sharp dispersion of the implants implying a sharp dispertion of the pn-junction capacitances (at least at the level of accuracy we are able to achieve). The distribution of the baseline is broad so that the high-end of each pixel for the same integrated flux will be in different part of the electrical transfer function. It is the root cause of the dispersion observed for non corrected ramps compare with the sharp dispersion observed for the corrected data.

Various strategies of generating the correction function of the pn-junction non linearity are under study. First method is to use the 200 last frames, where capacitive quadratic term dominates the trapping effects, to fit the $\mathrm{e} / \mathrm{V}$ correction. An illumination with a flux of $160 \mathrm{e} / \mathrm{s}$ covering the full dynamical range of the ADC has been used to fit the quadratic function. This is the most straightforward and pragmatic method which works with the expected accuracy but with a risk of keeping a small contribution of trapping or de-trapping.

Another method, which is under study, is to use two others reset voltages with the reset switch turn off to fit the e/V correction. With these runs a non-linearity is extracted from data but it is difficult to unambiguously correlate this non-linearity in the voltage domain with the one observed and measured in Figure 10 (left panel). It might be due to the contribution of parasitic capacitances before and after the reset of such three transistors circuitry. Algebraically it should be manageable even if the design of the pixel is unknown. This method is under 

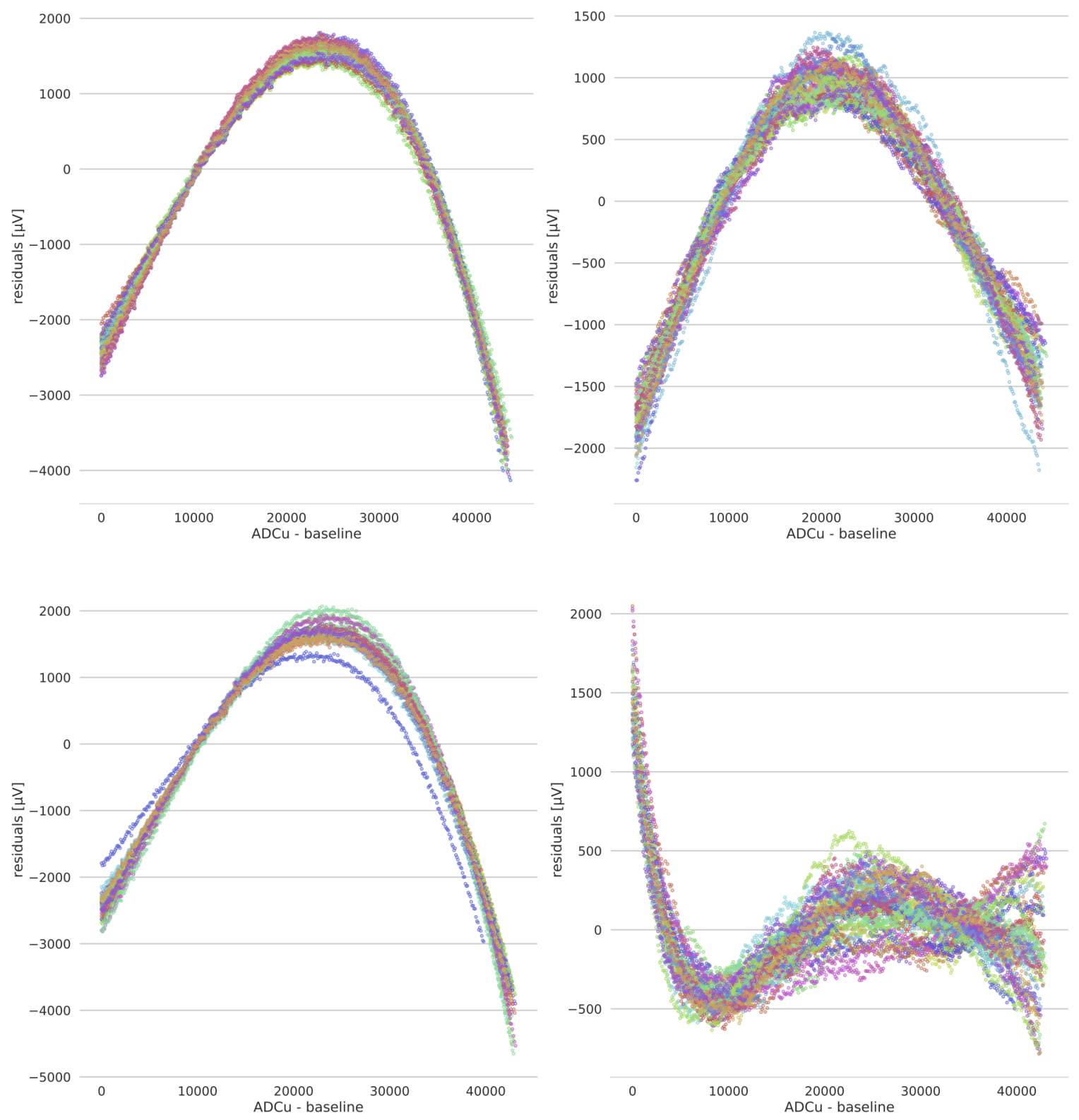

Figure 10. Quadratic behaviour due to non-linearity of the pn-junction e/V non-linearity for a 160 e/s flux with a moderate trapping in the second part of the ramp. This part, dominated by e/V non-linearity, is used to fit a quadratic correction corresponding to the non-linearity of the pn-junction under illumination

development since it could definitely decorrelate de-trapping/trapping contributions to the electrical non-linearity independently of the illumination history.

To illustrate the level of control that one able to achieve by applying a quadratic e/V transfer function, we show in Figure 11 the residuals of the fit of the flux applied only on the second part (high-end) of the data corrected by both $\mathrm{V} / \mathrm{V}$ and $\mathrm{e} / \mathrm{V}$ functions. The results are shown for several values of fluxes in the range expected in science observation. We notice that at the beginning of the ramp, the contribution of the trapping to the non-linearity increases with the absolute value of the flux. This linear increase in the zero point tends to tell us that, in first approximation, the trapping rate is constant for the low illumination range. This is not 
true for high fluxes (120-160 e/s). This feature has to be included in a trapping and de-trapping model which depends on the extension of the depletion volume. ${ }^{9}$

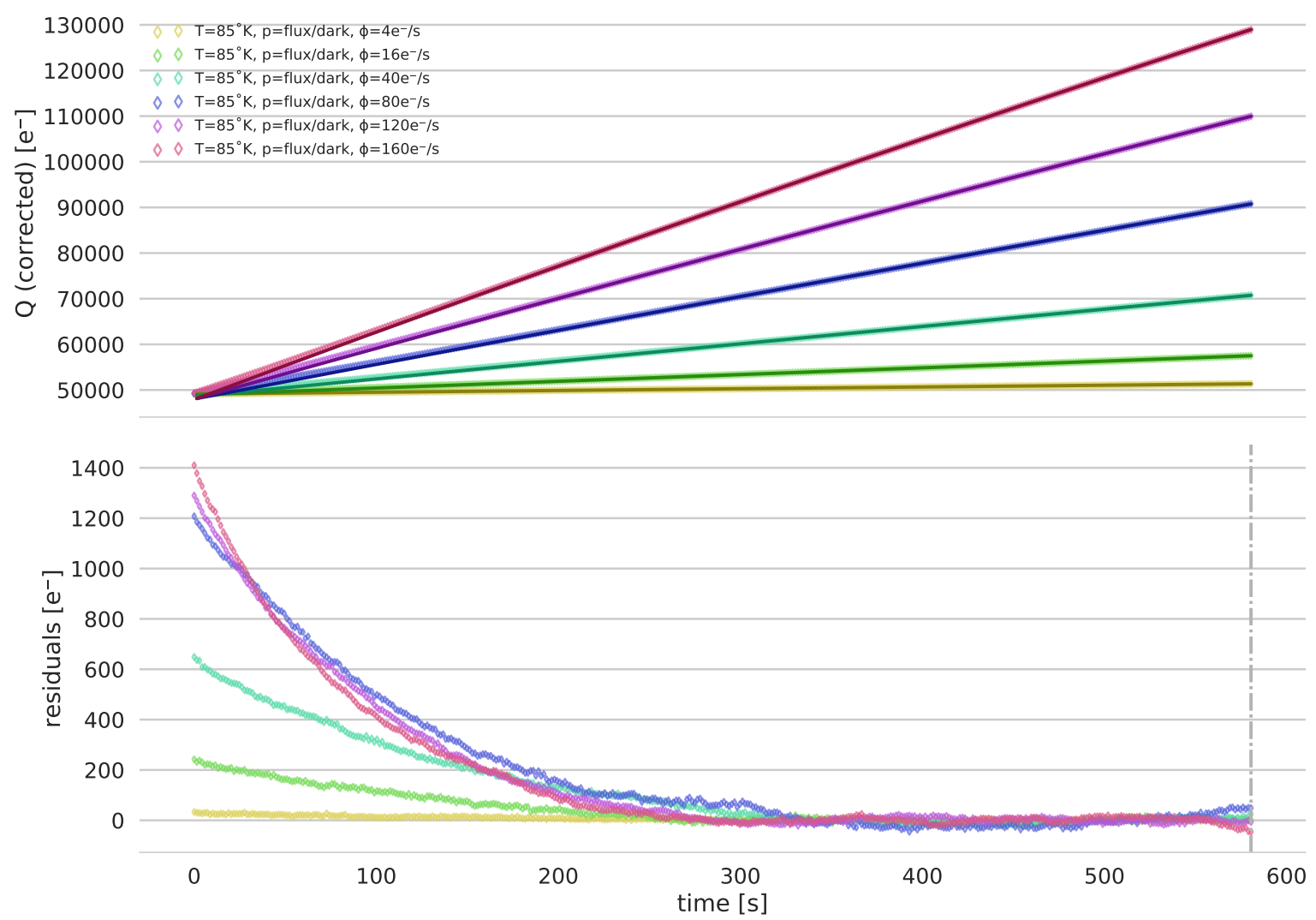

Figure 11. Top: slope corrected with e/V and V/V functions for various illumination intensities. The residuals to a linear fit show an increase of non-linearity due to trapping

\subsubsection{Trapping and de-trapping contributions to the non-linearity}

It was shown in section 4.2.2 that we are able to disentangle the trapping and de-trapping effects in the pixel response and the electrical $(\mathrm{e} / \mathrm{V}$ and $\mathrm{V} / \mathrm{V})$ non-linearity contributions. This gives us the possibility to study systematically de-trapping/trapping models varying the number of trap types, the total number of traps, the trapping and de-trapping rates, the densities of traps, the number of free and occupied traps as a function of the illumination history. This work is at its beginning and the results shown in the following sections of this paper are for illustration purposes only. Dispersion of the fitted parameters for a large sample of pixels has not been yet computed. Such effort requires a important software development work. Nevertheless, we observed that for some pixels the fit does not converge properly.

A simple example of trapping and de-trapping model: two types of traps The first attempt is to verify if a unique model of trapping and de-trapping is able to cover a large spectrum of illumination histories with a broad range of illumination levels (from 2 to $240 \mathrm{e} / \mathrm{s}$ ) and different types of patterns: (flux + short dark), (flux+long dark), (flux+zodiacal background), (flux+flux). The simple model of current versus time with trapping and de-trapping of charge carriers by two types of traps is defined by:

$$
\frac{\mathrm{d} q(t)}{\mathrm{d} t}=I_{q}(t)=\phi_{q}-\sum_{(x)}\left[\phi_{q} a_{0}^{(x)} \sigma_{a^{(x)}}+\left(A^{(x)}-a_{0}^{(x)}\right) \sigma_{\bar{a}^{(x)}}\right] e^{-\left(\phi_{q} \sigma_{a^{(x)}}+\sigma_{\bar{a}(x)}\right) t}
$$


where $q(t)$ is the charge carrier at time $t, \phi_{q}$ the photo-conversion current or the dark current, $a_{0}(x)$ the number of free traps at $t=0$ of type $(x)$ (with $x=0,1,2, \ldots), A^{(x)}$ the total number of traps of type $(x), \sigma_{a(x)}$ the probability that a free trap of type $(x)$ to capture a carrier, $\sigma_{\bar{a}^{(x)}}$ the de-trapping rate of occupied traps $\bar{a}^{(x)}$ of type $(x)$.

Results on the fit of the pixel $(2000,1063)$ from FR2 SCS 18628 are presented. Figures 13 to 15 (top panels) show for this pixel the $\mathrm{V} / \mathrm{V}$ and e/V corrected CDS (points) and the corresponding fitted model (lines) of the ramps for four illumination patterns: (flux + short dark), (flux+long dark), (flux+zodiacal background), (flux+flux). The residuals of the fit with a model of trapping/de-trapping with two types of traps are plotted in the bottom of each panels. Illumination pattern is reproduced many times, therefore we can consider that, in a circular way, each ramp is the preceding or consecutive ramp of the other. This circularity condition can be used to compute a mean ramp over iterations of the patterns (typically 20 iterations). The temporal evolution can also be fitted since the time stamp of each frame as well the time stamp of the status of the illumination are recorded. Various scenarios of very long illumination history can be study per pixel. This solution is kept for future work since it requires a huge effort on the development High Performance Computing (HPC).

For all of them the same model is used with two types of traps $(\mathrm{x}=0,1)$. For each illumination patterns the mean ramp is generated by averaging over the iteration of the same pattern. The residuals for all illumination patterns are within $+/-5 \mathrm{e} / \mathrm{s}$ without any temporal structure.

A strategy to combine into the same fit all informations coming from all types of patterns and fluxes is under development for the pixel calibration pipeline.

Trapping and de-trapping for the two different latency areas Figure 12 shows the trapping behaviour for two pixels belonging into two different latency areas shown in Figure 4. A fair difference is observed in the non-linearity of the pixel response for the first 150s of the ramp with illumination. 

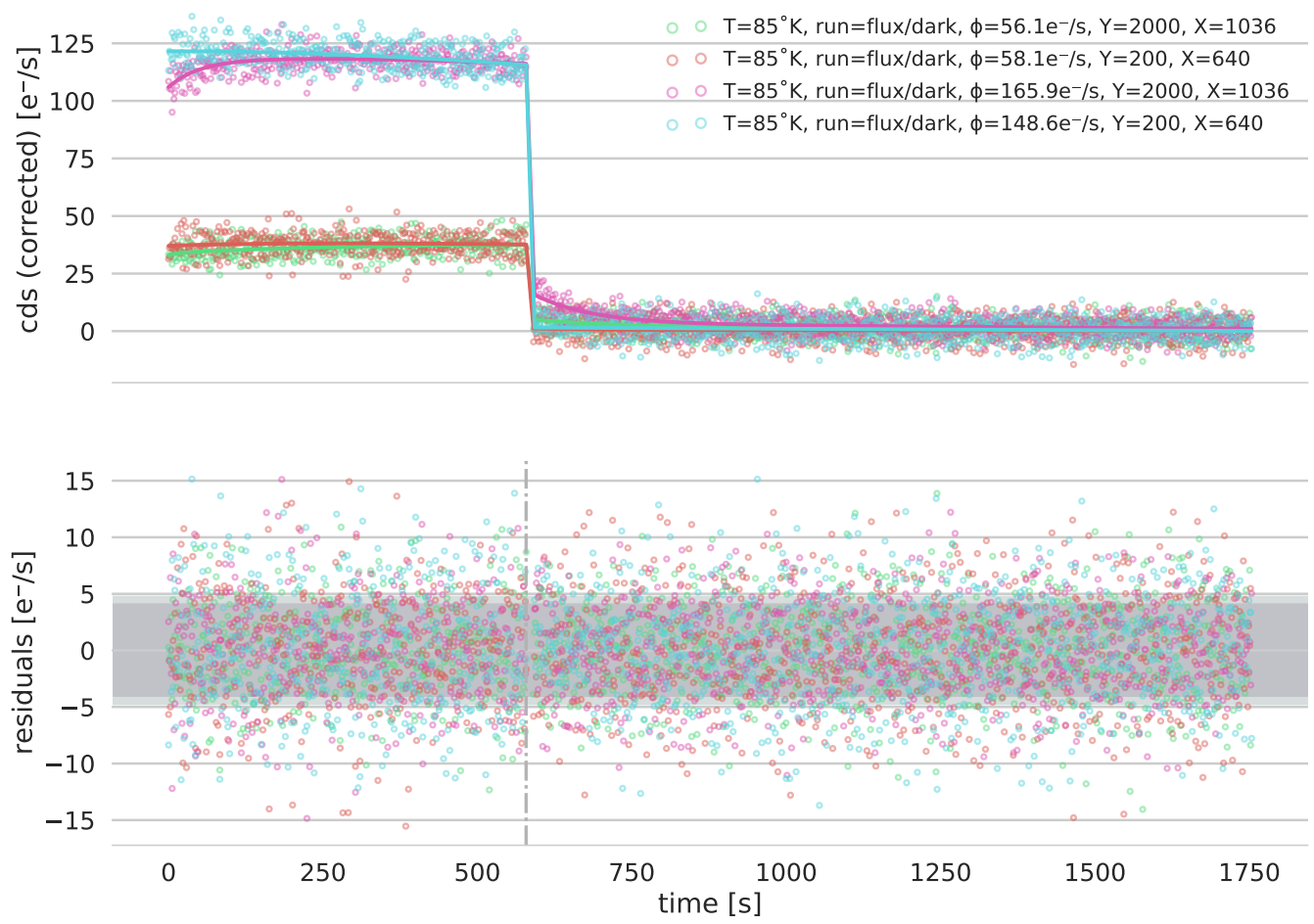

Figure 12. Trapping behaviour of two pixels taken from the two different latency areas shown in Figure 4. The configured flux are 57 and $160 \mathrm{e}-/ \mathrm{s}$ and the pattern is (flux + short dark).

Trapping and de-trapping versus the illumination pattern Figure 13 shows the same illumination flux followed or preceded by 3 different illumination states: a short dark which allows de-trapping, a zodiacal illumination of $2 \mathrm{e} / \mathrm{s}$ or an illumination with the same duration and intensity. Two different behaviours are observed at the beginning of the ramps (100 first frames). CDS increases due to decraising of the trapping in the (dark+zodiacal background) acquisition pattern while it decreases in (flux + flux) acquisition pattern where the de-trapping component is dominant.

The first remark is that the model is able to fit both patterns with trapping and de-trapping with residuals showing no systematics in their temporal structure. This is of the first importance for measuring the true flux value. 

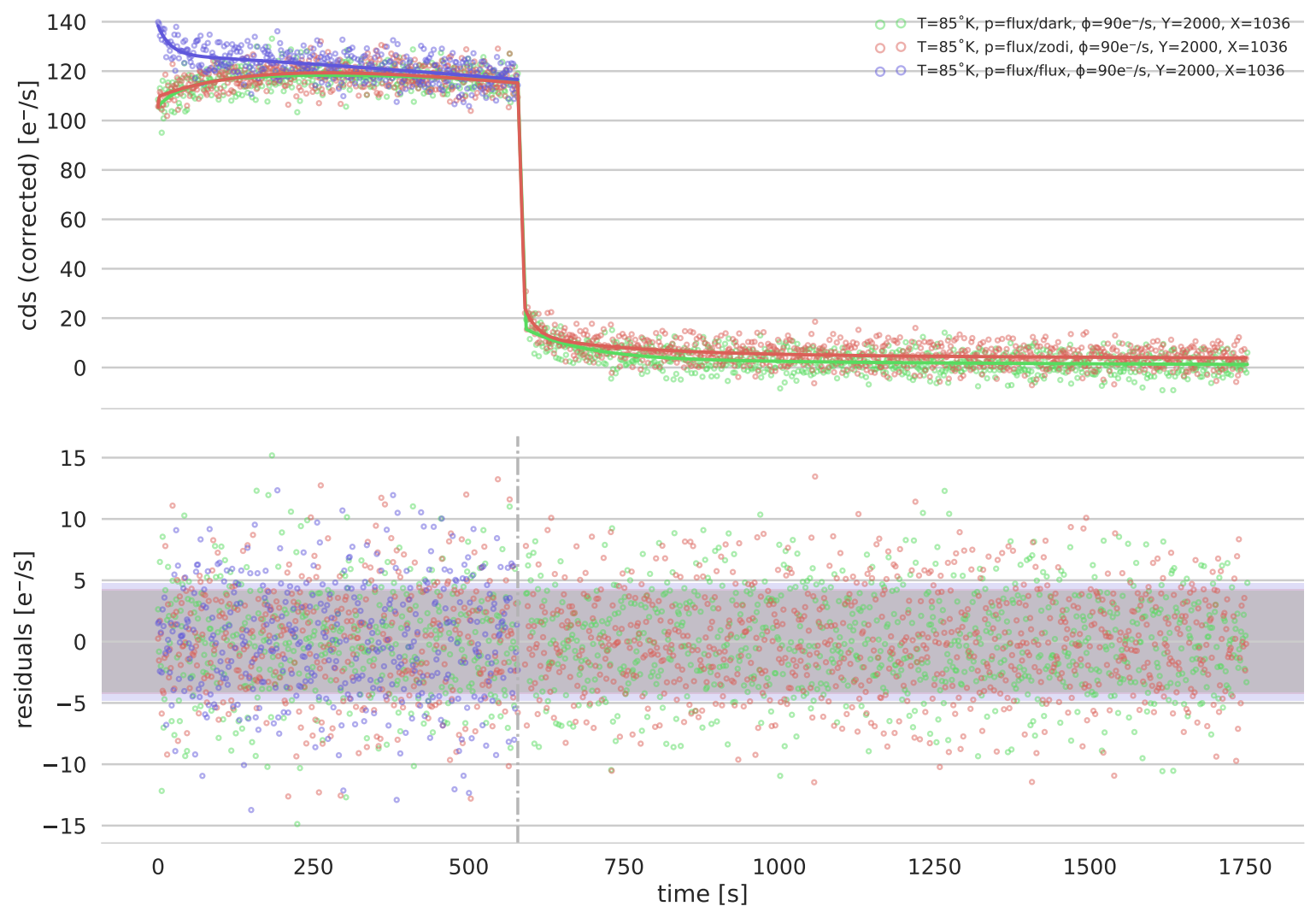

Figure 13. Trapping behaviour for pixel $(2000,1063)$ for a flux of 120 e/s and three illumination patterns: (flux+dark), (flux+zod. bkg) and (flux+flux)

Trapping and de-trapping versus the illumination levels for (flux + short dark) pattern Figure 14 shows the fit on different fluxes in the full dynamical range. Again the distribution of the residuals shows no structure at least within the statistical error. 


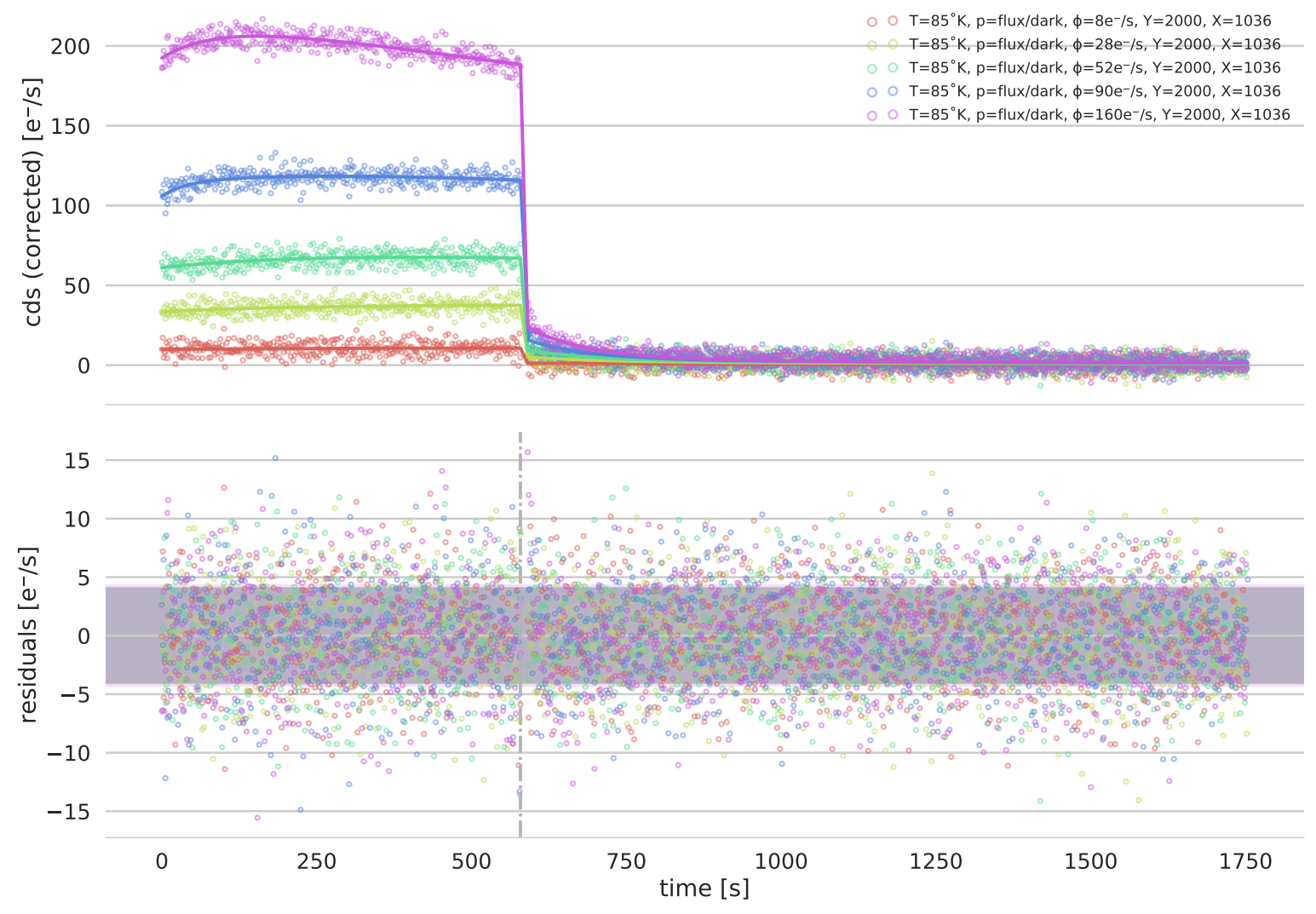

Figure 14. Trapping behaviour for pixel $(2000,1063)$ for different fluxes and the acquisition pattern: (flux + short dark)

Trapping and de-trapping observed with the (flux + long dark) illumination pattern for slow decay time component measurement Figure 15 shows a typical example of long dark for slow decay time component measurement. 

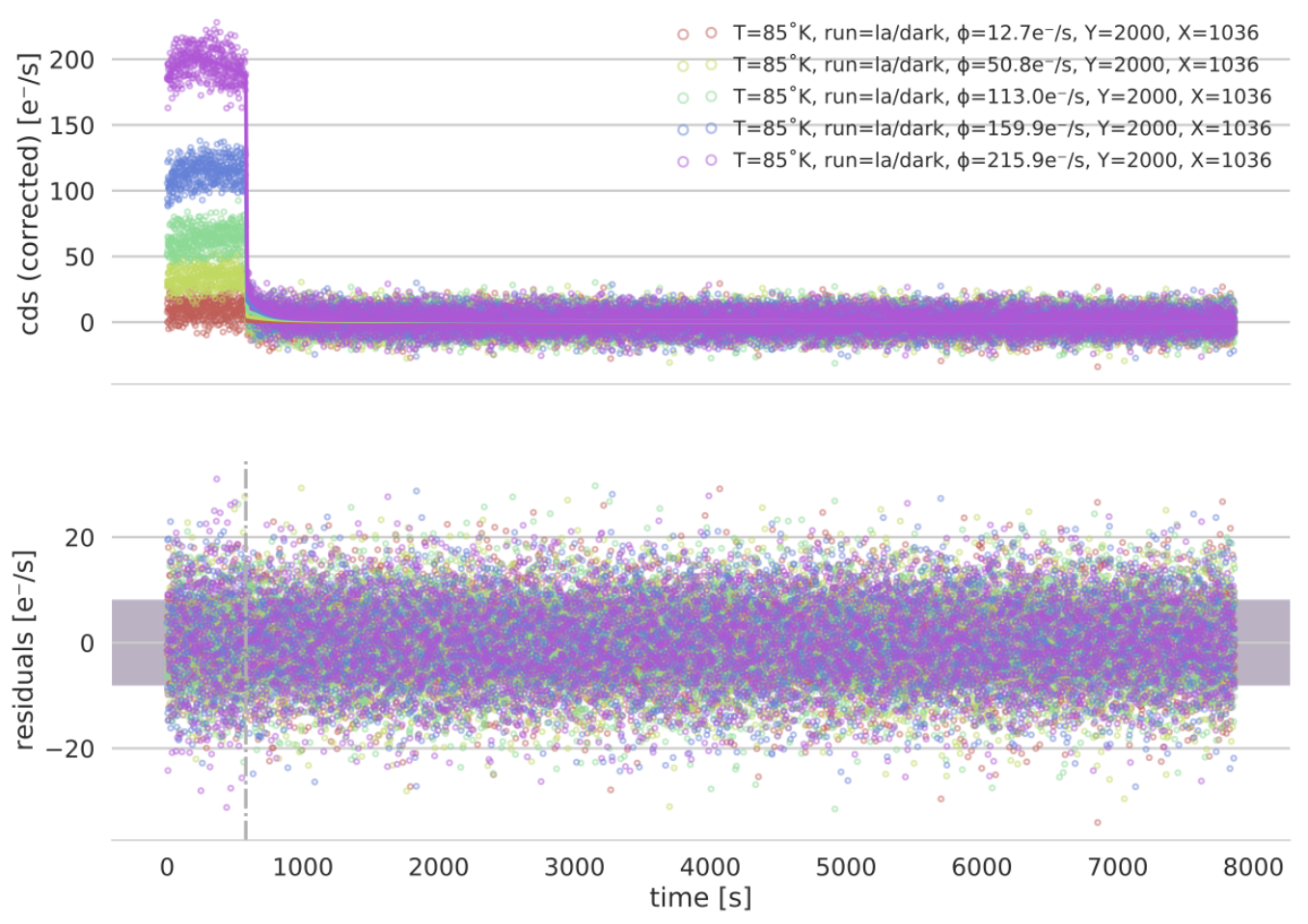

Figure 15. Trapping behaviour for pixel $(2000,1063)$ at $85 \mathrm{~K}$ for acquisition pattern (flux + long dark) for slow decay time constant analysis.

\section{CONCLUSION}

This work presented the pixel response calibration strategy of the NISP H2RG detector chains. The requirements on the calibration of the pixel response with an error budget has been introduced. It justified the test flow developed for the ground characterization of the SCS. The success of the characterization campaign has been demonstrated. The rationale for the measurements has been proposed and described in the context of the NISP characterization facility at CPPM. The acquisition sequences with various pattern of illumination and intensities have been described and justified.

The discussion on the results has been focused on one of the most difficult task of the pixel response calibration pipeline. The trapping/de-trapping contributions to the non-linearity measurement has been raised and illustrated with Flight Run data.

A tentative to use correction functions both in the charge and voltage domains (e/V and $\mathrm{V} / \mathrm{V})$ of the pixel response has been proposed and illustrated.

Encouraging results on Flight Run data have shown that the tuning of various models of trapping/de-trapping could be used to develop a pixel response calibration pipeline as a function of the illumination history. This work is just at this starting point and we expect more than 2 years of work on data analysis, detector physics and software coding of the pipeline to reduce more than $400 \mathrm{~TB}$ of data.

\section{ACKNOWLEDGMENTS}

This project is funded by CNES and ESA. We would like to thanks the Detector Characterization Lab of the NASA Goddard Spatial Flight Center and the Euclid detector team of the NASA, Jet Propulsion Laboratory for 
the fruitful discussions about the NASA acceptance test results in the framework of the NIR Detector Working Group which is a joint collaborative effort between NASA, ESA and Euclid Consortium.

\section{REFERENCES}

[1] Racca, G. et al., "The euclid mission design," Proc. SPIE 9904 (2016).

[2] "Teledyne imaging sensors, hawaii-2rg technical manual." MAN-0001Rev 4, 2009-06-05.

[3] "Teledyne imaging sensors, sidecar asic technical manual." MAN-0013 Ver 1.0, 2009-07-20.

[4] Secroun, A. et al., "Flight h2rg ir detectors: on-ground characterization for the euclid nisp instrument," Proc. SPIE 10709-78 (2018).

[5] Kohley, R. et al., "Random telegraph signal (rts) in the euclid ir h2rgs," Proc. SPIE 10709-53 (2018).

[6] Kubik, B., Barbier, R., Chabanat, E., Chapon, A., Clemens, J.-C., Ealet, A., Ferriol, S., Gillard, W., Secroun, A., Serra, B., Smadja, G., and Tilquin, A., "A new signal estimator from the nir detectors of the euclid mission," Publications of the Astronomical Society of the Pacific 128(968), 104504 (2016).

[7] Kubik, B., Barbier, R., Castera, A., Chabanat, E., Ferriol, S., and Smadja, G., "Impact of noise covariance and nonlinearities in nir h2rg detectors," in [Proceedings of SPIE - The International Society for Optical Engineering], 9154, 91541Q (07 2014).

[8] Simon M. Sze, M.-K. L., [Semiconductor Devices: Physics and Technology], John Wiley and Sons, Singapore Pte. Limited (August 2012).

[9] Smith, R. M., Zavodny, M., Rahmer, G., and Bonati, M., "A theory for image persistence in hgcdte photodiodes," Proc.SPIE 7021, 12 (2008). 